\title{
Clinical aspects of motor neurone disease
}

Richard W Orrell

Roberto J Guiloff

Richard W Orrell BSc MB ChB MD DCH FRCP FAAN is Associate Professor in Clinical Neuroscience at UCL Queen Square Institute of Neurology, and Honorary Consultant Neurologist at the National Hospital for Neurology and Neurosurgery, and Royal Free Hospital, London, UK. He has clinical and research expertise in motor neurone disease, and other neuromuscular and neurodegenerative diseases. Competing interests: Dr Orrell has received a fee for speaking from Biogen. He has acted as an expert witness related to motor neurone disease, and participates in clinical trials related to motor neurone disease. He has received fees from NICE for consulting.

Roberto J Guiloff LMSSA LPhil MD FRCP is Professor of Neurology and Emeritus Honorary Consultant Neurologist at Faculty of Medicine, University of Chile and Charing Cross Hospital, Imperial College Healthcare NHS Trust, London, UK and. He has clinical and research expertise in clinical trials, motor neurone disease and other neuromuscular diseases. Competing interests: none declared.

\begin{abstract}
Motor neurone disease (MND), or amyotrophic lateral sclerosis (ALS), is a progressive, fatal neurodegenerative disease affecting upper and lower motor neurones. It eventually causes widespread weakness and wasting, spasticity, dysarthria, dysphonia, dysphagia and respiratory failure. The diagnosis is clinical, and it is important for management to establish this early. It presents with upper and lower motor neurone signs affecting the bulbar, cervicothoracic and lumbosacral regions (ALS), only lower motor neurone signs affecting one or more limbs (progressive muscular atrophy), purely bulbar region symptoms and signs (bulbar onset), or only upper motor neurone signs in the (usually lower) limbs (primary lateral sclerosis). Imaging, electrophysiological and other tests help the differential diagnosis of mimics, including potentially treatable conditions. The underlying cause remains uncertain. Only $5-10 \%$ of patients have a demonstrable genetic cause. There is no cure, and the greatest advances have been in improving multidisciplinary supportive management. This includes gastrostomy and nutritional support, respiratory monitoring and management, including provision of non-invasive ventilation, and communication aids. Drugs for symptomatic relief of excessive salivation and spasticity can help. Riluzole and edaravone (restricted acceptance) are used although their disease-modifying effects are said to be mild and are controversial. Genetic therapies are currently being trialled for C9orf72- and SOD1-related MND/ALS.
\end{abstract}

\section{Keywords}

Amyotrophic lateral sclerosis; diagnosis; genetics; motor neurone disease; riluzole; spinal muscular atrophy; treatment 


\section{Key points}

- Motor neurone disease, also known as amyotrophic lateral sclerosis, is a progressive, fatal degenerative disease affecting upper and lower motor neurones

- The diagnosis is clinical, based on progressive denervation in muscles and pyramidal signs. These cause wasting, weakness and spasticity in bulbar, cervicothoracic and lumbosacral regions, in varying combinations, that cannot be explained topographically by a single lesion or other causes

- Differential diagnosis includes inflammatory motor neuropathies, mimics with combined upper and lower motor neurone signs (e.g. combined spinal cord and multiple root involvement in degenerative spine disease), spinal muscular atrophies and muscle diseases (e.g. polymyositis and inclusion body myositis)

- Established risk factors are older age, family history of motor neurone disease and male sex Around $5-10 \%$ of patients have an identified genetic cause, usually with autosomal dominant inheritance, although with incomplete age-dependent penetrance. The most frequent genetic causes relate to C9orf72 and SOD1

- Treatment is palliative multidisciplinary care, including speech, feeding and respiratory support and daily life adaptations

\section{Definition and incidence}

MND, also called amyotrophic lateral sclerosis (ALS), is progressive and fatal, usually within 4 years. Degeneration of both upper and lower motor neurones causes the key pathology and clinical signs. The cause is unknown. The incidence is 1.5 per 100,000 per year, and the prevalence 6 per 100,000 population. Approximately $50 \%$ of individuals die within $3-4$ years, $20 \%$ live $>5$ years, $10 \%>10$ years, and occasional patients 20 or more years. Risk factors for ALS are increasing age, a family history of ALS and male sex. MND affects individuals of all countries and racial backgrounds

The $5-10 \%$ of patients with a family history of ALS are referred to as having familial ALS, and those without a family history as having sporadic ALS. A small proportion of 'sporadic' cases show the same genetic changes as familial ALS.

\section{Pathogenesis}

The pathogenesis of ALS is uncertain, but a key pathological feature is TDP43 (TARDBP; TAR DNA binding protein)-containing inclusions in the motor neurone cytoplasm. Aggregates of disease-causing proteins could disturb normal protein homeostasis. Oxidative damage, intracellular mitochondrial dysfunction, glial cell pathology, glutamate excitotoxicity and defects in axonal transport, growth factors and RNA metabolism may also be involved.

Over 20 genes have been recognized in patients with ALS. Inheritance is usually autosomal dominant. A gene mutation is identified in around $75 \%$ of patients with a family history of ALS, and around $10 \%$ of apparently sporadic ALS. The most frequent gene abnormality in familial ALS $(40 \%)$ is a GGGGCC hexanucleotide repeat expansion in C9orf72; this is also found in patients with frontotemporal dementia (FTD) without ALS $-25 \%$ of patients with familial FTD, and $6 \%$ of 'sporadic' FTD. Mutations in the copper zinc superoxide dismutase gene (SOD1) (20\%), first identified in 1993, and $\operatorname{TARDBP}(5 \%)$, and $(5 \%)$ follow. The causative mechanism of the mutations remains uncertain. However, about lmost $90 \%$ of patients diagnosed with ALS do not have these gene changes. ${ }^{1}$

\section{Clinical features}

Essential clinical features are upper and lower motor neurone symptoms and signs, a progressive course and a lack of sensory involvement; however, some patients have sensory symptoms or changes on investigation. Symptoms and signs can start in the legs, arms or bulbar muscles, around one third in each region. A smaller proportion involves-have a respiratory presentation. The motor signs are asymmetrical and do not follow a nerve or root distribution.

Upper and lower motor neurone features are summarized in Table 1.There is a wide range of clinical presentations, especially in the early stages

Amyotrophic lateral sclerosis - both upper and lower motor neurone signs are present. Typical features are foot drop, hand-wasting and weakness, and/or speech and swallowing difficulties. (Figure 1).

Progressive muscular atrophy - as it only has lower motor neurone features that can obscure upper motor neurone signs, this can be confused with spinal muscular atrophy (SMA). 
Progressive bulbar palsy - this presents with dysarthria and/or dysphagia caused by upper (pseudobulbar palsy), lower or upper plus lower motor neurone signs. If seen in isolation, brainstem or brain pathology must be ruled out (Figure 1, Table 1)

Primary lateral sclerosis - only upper motor neurone features are present. If denervation is not evident after 5 years, a diagnosis of primary lateral sclerosis can be made, with a much better prognosis.

Cognitive dysfunction is variable and may appear as behavioural alteration. There is an overlap between ALS and FTD.

\section{Diagnosis}

The diagnosis should only be made clinically, by close attention to the history, examination findings and clinical interpretation of investigation results. Over-reliance on investigations can be misleading. Expert clinical examination is usually sufficient to define the presence, nature and extent of upper and lower motor neurone involvement.

Electromyography (EMG) and nerve conduction studies (NCSs) - EMG may identify denervation in muscles of regions with early or no clinical involvement. NCSs exclude motor and other polyneuropathies (particularly multifocal motor polyneuropathy).

Central motor conduction time - measurement may identify corticospinal tract involvement but is very rarely useful or needed. involvement:

Other investigations eliminate other possible causes of upper and lower motor neurone - Magnetic resonance imaging of brain and spinal cord helps to exclude pathology in the brain, spinal cord and roots.

-Cerebrospinal fluid (CSF) examination is not required for diagnosing ALS but may exclude other conditions. In ALS the CSF is normal except that $20 \%$ of patients show protein increases of up to 1.5 g/litre.

-Blood tests are not diagnostic but may determine other causes of treatable neuropathy or myelopathy.

-Muscle biopsy is not needed to diagnose ALS but can be performed if there is concern of a myopathic condition.

-Genetic testing, in terms of DNA analysis, is currently not usually considered a diagnostic investigation for ALS.

\section{Differential diagnosis}

Multifocal motor neuropathy can mimic a lower motor neurone presentation of ALS. NCSs demonstrate focal conduction blocks related to demyelination. It can be treated with intravenous immunoglobulin.

Cervical and lumbosacral degenerative disease with disc spinal cord compression in the cervical region and multiple cervical and lumbosacral radiculopathies can give combined pyramidal signs, limb wasting and weakness, show progression and be helped by surgery.

Inclusion body myositis can be confused with a lower motor neurone presentation of ALS.

Primary inflammatory myopathies can be suggested by raised serum creatine kinase and evidence of mixed myopathic and active denervation features on EMG.

Important differential diagnoses of ALS include other diseases that affect lower but not upper motor neurones:

-Spinobulbar muscular atrophy (SBMA), or Kennedy's disease is an X-linked lower motor neurone disease of male patients; there is gynaecomastia, diabetes mellitus, infertility and abnormal sural sensory action potentials.

-SMA shows symmetrical clinical features in the classical forms (types 1, 2 and 3). Type 1 presents around birth, and types 2 and 3 later in childhood, adolescence and adulthood.

\section{Management}

Many patients miss out on early support and therapy for the condition because of a delay in diagnosis. $^{2}$

\section{Disease-modifying therapy}

Riluzole is recommended by the UK National Institute for Health and Care Excellence (NICE) ${ }^{2}$, although its benefit in slowing progression of MND/ALS is controversial. ${ }^{3}$ Edaravone is used in many countries but is not available in the UK. ${ }^{56}$ 


\section{Symptomatic therapies ${ }^{54}$}

Management of patients with ALS involves a coordinated multidisciplinary palliative care approach that meets the changing physical and psychosocial needs of patients, families and carers throughout the disease course. Physical, occupational, and speech and language therapies are important components (Figure 2).

Dysphagia and nutrition: in the early stages, nutritional supplements can be helpful. Percutaneous endoscopic gastrostomy or radiologically inserted gastrostomy is used for nutritional and food support when feeding is significantly disrupted, with danger of aspiration or loss of weight. ${ }^{4}$ Complications of these procedures are more likely if there is respiratory involvement.

Dysarthria: assessment of the clinical features of dysarthria by the neurologist and speech and language therapist determines the management of communication deficits. This varies from antispastic agents for spastic dysarthrias, and speech therapy techniques for mild dysarthrias, to simple aids for written communication and computerized devices with speech production. ${ }^{65}$

Salivary problems: sialorrhoea is a major problem in patients with advanced disease.

Anticholinergics, including hyoscine patches and atropine, and drugs with anticholinergic action such as amitriptyline and glycopyrrolate, can help. Botulinum toxin injection of the salivary glands may need to be repeated every 3 months. A suction device can also be provided.

Respiratory failure: this is the most frequent cause of death in ALS. It relates to denervation and weakness of the diaphragmatic, intercostal and accessory muscles of respiration, worsened by the likelihood of aspiration pneumonia as a result of bulbar palsy. An expectant management approach dependent on patient preference is preferred. Non-invasive respiratory support or ventilation is increasingly used, with the goal of reducing symptoms related to nocturnal hypoventilation (morning headache, fatigue), and later respiratory distress from respiratory insufficiency ${ }^{4}$

The need for invasive ventilation (tracheostomy) can often be discussed in advance. Management of patients with tracheostomy and ventilation is demanding for community services, patient and family, and many patients decide not to proceed. Other patients have prolonged survival with tracheostomy and home ventilation (Figure 3).

Pain, cramps and spasticity: immobility, loss of muscle, and general debility can lead to pain. Analgesics and non-steroidal agents should always be tried first. Oral morphine or transdermal fentanyl patches can help in severe cases but caution is needed if respiratory function is poor. With spasticity, baclofen, dantrolene and tizanidine can be used and physiotherapy may be helpful. Quinine sulphate is given for cramps.

Advance care-planning and emotional support: advance care-planning is important. This includes decisions on how to proceed, with or without gastrostomy, non-invasive ventilation or tracheostomy, and what to do in the event of severe illness such as chest infection. It is important that any advance care plan is known to relatives, carers, therapists and medical staff.

Depression can be a normal reaction to the diagnosis and situation. However, It can also be a manifestation of the neurodegenerative process, as can emotional liability.

Genetic advice: this is well established for patients with SMA and SBMA. Advice is more complex for ALS as a range of genes is involved, penetrance is incomplete and the genetic mechanism is not clear.

\section{Future developments}

The most significant recent advances in clinical care of ALS have been multidisciplinary team involvement and agreed management guidelines.

The prognosis of childhood-onset (type 1) SMA has recently changed dramatically. Two gene therapies reverse the disease process here, and babies who would otherwise have died may now develop and progress to walking. Both therapies are approved by the US Food and Drug Administration, and one by NICE in the UK. It remains to be seen how this might translate to ALS, where the disease processes are not clear. Clinical trials in genetic forms of ALS including C9Oorf72and SOD1-related disease are underway. 


\section{KEY REFERENCES}

1. Morgan S, Orrell RW. Pathogenesis of amyotrophic lateral sclerosis. British Medical Bulletin 2016; 119: 87-97.

2. National Institute for Health and Care Excellence. Motor neurone disease: assessment and management. NG42. 2019. www.nice.org.uk/guidance/ng42 (accessed 14 May 2020.

3. Sandercock J, Burls A, Hyde C, et al. Riluzole for motorneuron disease. More trials are needed. BMJ 2001; 322: 1305.

4. Andersen PM, Abrahams S, Borasio GD, et al. EFNS guidelines on clinical management of amyotrophic lateral sclerosis (MALS) - revised report of an EFNS workforce. Eur J Neurol 2012; 19: 360-75.

5. Tomic B, Guiloff RJ. Dysarthria in amyotrophic lateral sclerosis. A review. Amyotroph Lateral Scler 2010; 11: 4-15.

\section{Further reading}

6Jackson C, Heiman-Patterson T, Kittrell P, et al. Radicava (edavarone) for amyotrophic lateral sclerosis: US experience at 1 year after launch. Amyotroph Lateral Scler Frontotemporal Degen 2019; 20: $605-10$.

\section{Legends}

Figure $1 \mathrm{~A}$ patient with MND, illustrating wasting and weakness in the muscles of the limbs and tongue.

Figure 2 Multidisciplinary care of MND.

Figure $3 \mathrm{~A}$ patient requiring full support, including gastrostomy, tracheostomy ventilation, and eye gaze communication device.

Table 1.

Upper and lower motorneuron signs by regional symptoms (adapted from reference 65 ). 


\section{TEST YOURSELF}

To test your knowledge based on the article you have just read, please complete the questions below. The answers can be found at the end of the issue or online here.

\section{Question 1}

A 56-year-old man presented with a 1-year history of progressive difficulty walking and cramps in the legs, needing a stick, slurred slow speech and difficulty with feeding, which was taking too long. Drinking fluids occasionally made him cough. His weight was stable.

On clinical examination, he had laboured mastication and difficulty swallowing, slow tongue movements, tongue fasciculations, poor palatal movement and brisk gag and jaw reflexes. In the upper limbs biceps and triceps jerks were very brisk. There was also wasting, weakness (Medical Research Council grade 4) and fasciculations in the right leg affecting the quadriceps and tibialis anterior, along with weakness of left hip flexion and foot dorsiflexion, with spasticity. The right knee jerk was brisk. The left knee jerk and ankle jerk were very brisk. Both plantar responses were weakly flexor. There were no abnormat sensory signs.

\section{Select one or more if any is, or are, correct answer as it applies to the above case}

\section{Diagnosis of MND/ALS}

A. In the bulbar region he has only lower motorneurone signs

B. In the upper limbs he has only upper motorneurone signs

C. In the right leg he has only lower motor neurone signs

D. He has upper and lower motor neurone signs in the bulbar region and left leg.

E. He has upper and lower motor neurone signs in the bulbar, cervical and lumbosacral regions

\section{Differential diagnosis}

A. Spinal Muscular Atrophy

B. Multifocal Motor Neuropathy

C. Primary Lateral Sclerosis

D. Brain stem tumour

E. None of the above

\section{Investigations (select the most useful on for diagnosis}

A. MRI thoracic region

B. Electromyography upper limbs

C. Genetic study

D. Cerebrospinal fluid

E. Creatine kinase in blood

\section{Management (select the most important)}

A. Inmediate PEG tube insertion

B. ivlg

C. C. Oral steroids

D. Full respiratory assessment

E. Speech therapy Referral to palliative care and end of life pathway

\section{Correct answers}

1, B correct 1A Has also upper motorneurone signs in the bulbar region (table 1) 1C. Has also brisk right knee jerk (table 1); 1D: he does not have lower motor neuron signs in the left leg $1 \mathrm{E}$ He does not have lower motor neurone signs in the cervical region (arms)

2E correct.. 2A SMA does not have upper motorneuron signs 2B Multifocal motor neuropathy does not have upper motorneurone signs. $2 \mathrm{C}$ Primary lateral sclerosis does not have lower motorneurone signs. 2E Brain stem tumor does not have lower motor neurone signs in the limbs3B Correct. To obtain evidence of denervation iin the cervical region which makes the diagnosis certain with both upper and lower motoneurone features in three regions and progression. 3A Does not add to the diagnosis $3 \mathrm{C}$. No family history, not required for diagnosis, only for possible cause. 3D Not required for diagnosis E. Not required for diagnosis 
4D correct: essential for decisions about indication and timing of management of dysphagia/peg tube insertion 4A No weight loss, only occasional cough when drinking, no prior assessments of respiratory function and dysphagia 4B and 4C Not indicated for MND; 4E Not as important for dysphagia assessments at presentation,

\title{
Question 2
}

A 30-year-old man presented with progressive weakness in the right hand. This prevented his work as a watchmaker.

On clinical examination, fasciculations were seen in the right forearm. There was preserved muscle bulk in the right thenar eminence. There was Medical Research Council grade 3 weakness in the right hand, especially of the right thumb, but also of finger abduction and flexion. Sensation was normal in the right hand. Reflexes were absent in the right arm, but normal in the left arm and legs.

\section{Investigations}

-Cerebrospinal fluid protein $1.1 \mathrm{~g} /$ litre $(0.15-0.45)$

-Nerve conduction studies showed conduction block in the right forearm, and normal sensory

conduction.

\author{
What is the best initial treatment for the patient? \\ A Riluzole \\ B Methylprednisolone intravenously \\ C Edaravone \\ D Immunoglobulin intravenous infusion \\ E Vitamins $\mathrm{C}$ and $\mathrm{E}$
}

\section{Correct answer: D.}

D correct. This is the preferred treatment for multifocal motor neuropathy (MMN). B

Methylprednisolone may cause deterioration in MMN. C Edaravone has not been demonstrated to be effective in MMN, but may be effective in MND. E. Vitamins $C$ and $E$, used as antioxidants, have not been demonstrated to be effective in MMN.

\section{Question 3}

\section{NEW QUESTION 3}

A 45-year-old man had been found to have motor neurone disease. Genetic testing showed that he was carrying the C9orf72 gene.

\section{What are the implications of this finding that should be discussed with him?}

Or

A 45-year-old man had been found to have motor neurone disease. His maternal grandfather had died early of a wasting disease but there were no further details.

What approach to genetic testing should be discussed with him? 